\title{
5 Research Square

\section{Development of a Decellularized Hypopharynx with Vascular Pedicle Scaffold for use in Reconstructing Hypopharynx}

Nan Hou ( $\nabla 110565888 @ q q . c o m)$

Chengdu Medical College

Die LV

Chengdu Medical College

XiaoLi Xu

Chengdu Medical College

YanQing Lu

Chengdu Medical College

JingZhi Li

Chengdu Medical College

RuiNa Ma

Tangdu Hospital, Fourth Military Medical University

Ying Tang

Chengdu Medical College

Yun Zheng

Sichuan University

\section{Research Article}

Keywords: decellularization, extracellular matrix, hypopharynx, vascular pedicle, hypopharyngeal squamous cell carcinoma

Posted Date: October 14th, 2021

DOl: https://doi.org/10.21203/rs.3.rs-960447/v1

License: (c) (i) This work is licensed under a Creative Commons Attribution 4.0 International License. Read Full License

Version of Record: A version of this preprint was published at Artificial Organs on February 22nd, 2022. See the published version at https://doi.org/10.1111/aor.14214. 


\section{Development of a decellularized hypopharynx with vascular pedicle scaffold for use in reconstructing hypopharynx}

Nan $\mathrm{Hou}^{\mathrm{a}, 1, *}$, Die Lva, b, 1, XiaoLi Xu ${ }^{\text {a, }}$, YanQing $\mathrm{Lu}^{\mathrm{a}}$, JingZhi $\mathrm{Li}^{\mathrm{a}}$, RuiNa $\mathrm{Ma}^{\mathrm{c}}$, YingTanga, YunZheng ${ }^{\mathrm{d}}$

${ }^{a}$ :Department of Otorhinolaryngology Head and Neck Surgery, Clinical Medical College and The First Affiliated Hospital, Chengdu Medical College, Chengdu City, Sichuan Province, China

b: Department of Otorhinolaryngology Head and Neck Surgery, Renshou People Hospital, Chengdu City, Sichuan Province, China

c: Department of Otorhinolaryngology Head and Neck Surgery, Tangdu Hospital, Fourth Military Medical University, Xi'an, City,Shaanxi Province, China

d: Department of Otorhinolaryngology Head and Neck Surgery, West China Hospital, Sichuan University, Chengdu City, Sichuan Province, China

*: Corresponding author.

Nan Hou: Tel: +8613678174505. E-mail addresses: 110565888@qq.com.

${ }^{1}:$ The authors contributed equally to this study.

Abstract: Background:Hypopharynx reconstruction after hypopharyngectomy is still a great challenge. Perfusion decellularization is for extracellular matrix (ECM) scaffolding and had been used in organ reconstruction. Our study aimed to prepare an acellular, natural, three-dimensional (3D) biological hypopharynx with vascular pedicle scaffold as the substitute materials to reconstruct hypopharynx.Result:Scanning electron microscope (SEM) and immuno showed that the decellularized hypopharynx with vascular pedicle scaffold retained intact native anatomical ECM structure. Myoblasts were observed on the recellularized scaffolds with bone marrow mesenchyml stem cells (BMSCs) induced by 5 -azacytidine implanted in the rabbit greater omentum by immunohistochemical analysis. Conclusion:the decellularized hypopharynx with vascular pedicle scaffold prepared by detergent perfusion in our study has an potential to be an alternative material to pharynx reconstruction.

Keywords: decellularization, extracellular matrix, hypopharynx, vascular pedicle, hypopharyngeal squamous cell carcinoma

\section{Introduction}

Hypopharyngeal squamous cell carcinoma (HSCC) accounts for approximately $3 \%$ to $5 \%$ of all head and neck squamous cell carcinomas (HNSCC), and is less prevalent than most other major HNSCC, such as the laryngeal cancer and oral squamous cell carcinomas $(1,2)$. However, these patients with HSCC have worse prognosis than other types of HNSCC since the tumor in these patients has developed into stage III or IV when diagnosed $(1,3,4)$. The current treatment methods of HSCC mainly include surgical resection and concurrent chemoradiotherapy (CRT). Hypopharynx refers to the muscular canal from the upper margin of hyoid bone to the lower margin of cricoid cartilage. The tumor may invade larynx, epiglottis or tongue, etc. The surgical resection of advanced hypopharyngeal cancer is so extensive that primary pharyngeal closure cannot complete directly or may lead to a high risk for pharyngocutaneous fistula postoperatively (5-8). Furthermore, the wide range of resection will lead to the loss of voice and swallowing function, which reduce the patients' life quality. Reconstruction is critical to maintain hypopharynx functions like voice and swallowing, as well as improve patient's life quality.

Hypopharynx reconstruction has been a great challenge. Recently, the common materials used to reconstruct hypopharynx are autogenous flaps, including pedicled flaps and free flaps. The 
pedicled flaps include pectoralis major pedicled flaps, supraclavicular artery flaps, and free flaps include radial forearm free flap, anterolateral thigh free flap, jejunal free flaps, temporomandibular free flaps, serratus anterior free flap, gracilis muscle free flap, etc (5, 9-19). It causes secondary injury to sick patients and is difficult to complete second repair when the autogenous flaps necrosed.

With the development of tissue engineering, a substantial number of biological materials were used in organ or tissue reconstruction including artificial and native biomaterials. Common artificial biomaterials included collagen, fibrin, silk, alginate and so on. However, these materials are lack of the structural, chemical, biological, and mechanical complexity of native ECM (20). The simple native biomaterials such as allogeneic acellular dermal matrix patch are only used to repair a small range of defects (21-23). In our experiment, the complex full-thickness ECM with vascular pedicle scaffold were prepared to solve the large scale of hypopharyngeal defects.

Decellularization techniques basically preserves all the characteristics of natural ECM and decellularized scaffold can provide a good micro-ecological environment for the growth, proliferation and differentiation of cells $(24,25)$. Ott et al. prepared a acellular, natural, three-dimensional (3D) biological cardiac scaffold retaining the natural structure by perfusion and decellularization, and successfully simulated cardiac tissue with a certain contraction function (26). They also decellularized rat, porcine, and human kidneys by perfusion, yielding acellular scaffolds with vascular, cortical and medullary architecture, collecting system and ureters and transplanted in orthotopic position in rat successfully (27). Moreover, it was reported that decellularized scaffolds for liver, pancreas, lung, heart, and mitral valve were prepared by decellularization techniques (28-36). The antegrade or retrograde perfusion can completely retain the vascular bed (26, 37, 38). Zhang, Jian, et al prepared decellularized rectus abdominis scaffold by decellularization techniques for repairing the defect of abdominal wall and the decellularized scaffold showed good neovascularization and functional regeneration ability (38). Previously we have prepared decellularized laryngeal scaffolds with preserved cartilage by the same method and induced myocytes successfully $(39,40)$. Repairing hypopharyngeal defects with vascularized flaps can reduce the incidence of postoperative pharyngocutaneous fistula, infection and other complications(41). However, there is no report using decellularized hypopharynx with vascular pedicle to solve the defect after hypopharyngectomy.

Therefore, a complex full-thickness native decellularized hypopharynx with vascular pedicle scaffold was prepared by perfusing the ascending pharyngeal artery with $1 \%$ SDS and triton X-100 in our experiment. And the results indicated that the composite scaffold retained intact native anatomical ECM structure and component by HE staining and SEM and low immunogenicity by immunofluorescence. In addition, we examined the regenerative myoblasts growing in the ECM by immunohistochemical analysis. We aimed to prepared a new alternative material for hypopharynx reconstruction.

\section{Materials and methods}

\subsection{Animals}

All rabbits used in the present study were obtained from the Chengdu Dashuo Biotechnology Co. LTD (Chengdu, China) and the research complied with all committee regulations approved by the Ethics Committee for Animal Experimentation of Clinical Medical College and The First Affiliated Hospital of Chengdu Medical College. Adult New Zealand White Rabbits $(n=36)$, 
weighing $2.5-3 \mathrm{~kg}$, were used as donor animals and randomly divided into 2 groups ( $\mathrm{n}=12$ each): (1) the perfusion group (decellularized hypopharynx with vascular pedicle), and (2) the control groups (fresh hypopharynx with vascular pedicle with no treatment). Two scaffolds were extracted per rabbit. The scaffolds ( $\mathrm{n}=3 /$ time/group) were observed by macroscopic visualization, histological examination, SEM, molecular analysis, and immunological assessment. The remaining animals $(n=3 /$ time/group) were implanted in the greater omenta of recipient rabbits who were anesthetized by intraperitoneal sodium pentobarbital administration $(40 \mathrm{mg} / \mathrm{kg})$. The implantation procedure and tests are described in detail below.

2.2 Purfusion decellularizion of hypopharynx with vascular pedicle

The adult New Zealand white rabbits were euthanized by intraperitoneal sodium pentobarbital administration $(100 \mathrm{mg} / \mathrm{kg})$. After systematic heparinization through the ear vein, a median incision of the neck exposed hypophargnx and trachea fully. The two common carotid arteries were dissected. Their superior and inferior extremities, and other branches were ligated, but their branches (superior thyroid arteries, superior laryngeal arteries and ascending pharyngeal artery) were preserved. One transfusion needle was inserted into the left common carotid arteries. The hypophargnx and common carotid arteries were removed and put on a super-clean bench. The perfusion sequence was in accordance with the Ott' method [26]. The hypophargnx was infused at a speed of $99 \mathrm{ml} / \mathrm{h}$ by a micropump (Guangxi Veryark Technology Co., Ltd., Guangxi,China) under aseptic conditions. Briefly, heparinized phosphate buffered saline (PBS, sigma) containing adenosine was perfused into the left carotid for $15 \mathrm{~min}$ and then $1 \%$ sodium dodecyl sulfate (SDS; Sigma) in deionized water was perfused for $16 \mathrm{~h}$. This was followed by perfusion with deionized water for $15 \mathrm{~min}$ and $1 \%$ Triton X-100 (Sigma) in deionized water for $30 \mathrm{~min}$. In the end, the hypophargnx was perfused with PBS containing $100 \mathrm{U} / \mathrm{ml}$ of penicillin-G (Sigma) and $100 \mathrm{U} / \mathrm{ml}$ of streptomycin (Sigma) for $48 \mathrm{~h}$. During the purfusion, we maintained the entire hypophargnx in RPMI-1640 culture media without serum in an aseptic culture flask.

\subsection{Characterization of decellularized scaffolds}

\subsubsection{Histological analysis}

The samples were fixed in 10\% formalin in PBS for $24 \mathrm{~h}$ at room temperature and then washed in distilled water, dehydrated in graded alcohol, embedded in paraffin, and sectioned. The sections were stained with hematoxylin and eosin (HE).

\subsubsection{Scanning Electron Microscope (SEM)}

The decellularized scaffolds were fixed with $2.5 \%$ glutaraldehyde in PBS for $24 \mathrm{~h}$ at $4{ }^{\circ} \mathrm{C}$. The samples were washed in PBS, dehydrated in graded alcohol, critical point-dried, and coated with gold. The samples were observed via SEM (Hitachi S-2500 Scanning Electron Microscope) to analyse the ultrastructure of the surface of pharyngeal constrictor and vessel.

\subsubsection{PCR determination of DNA content}

Three types of tissue samples were examined: fresh hypopharynx, decellularized hypopharynx, and decellularized vessel (about $25 \mathrm{mg}, \mathrm{n}=3$ for each sample). DNA was isolated with a commercially available extraction kit (Tiangen, Beijing, China). The total DNA content was measured by absorption at $260 \mathrm{~nm}$ on a UVeVIS spectrophotometer (Biorad, US). The total DNA was used as a template for polymerase chain reaction (PCR) analysis with primers specific for $\beta$-actin(F: 5'-CGAGATCGTGCGGGACAT-3' R: 5'-CAGGAaGGAGGGCTGGAAC-3'). PCR products were separated by electrophoresis in $1.5 \%$ agarose gels.

2.3.4 Immunohistochemical analysis 
The decellularized and fresh scaffold were fixed with 4\% paraformaldehyde in $0.1 \mathrm{M}$ PBS containing $30 \%$ sucrose at $4{ }^{\circ} \mathrm{C}$. Then the samples were cut to $10 \mathrm{~mm}$ sections on a freezing microtome (Leica), mounted on poly-L-lysine-coated slides, and washed in $0.1 \mathrm{M}$ sodium PBS with $0.25 \%$ Triton X-100 (PBS/Triton, $\mathrm{pH}$ 7.2). Subsequently, the pieces were incubated with rabbit anti-MHC class II Polyclonal antibody (Bioss, Beijing, China) $(1: 100)$ at $4^{\circ} \mathrm{C}$ for overnight. The pieces were then incubated with CoraLite488-Conjugated Affinipure goat anti-rabbit IgG $(\mathrm{H}+\mathrm{L})$ (Bioss, Beijing, China) (1: 300) for $30 \mathrm{~min}$ and washed twice in PBS. Finally, the samples were stained with DAPI for 3 min and washed with $0.1 \mathrm{M}$ PBS. All pieces were observed under a fluorescence microscope (Olympus).

2.3.5 Cytotoxicity of decellularized hypopharynx with vascular pedicle

5-mmdiameter decellularized composite scaffold samples were cultured in L-DMEM supplemented with $15 \%$ fetal bovine serum, $100 \mathrm{U} / \mathrm{ml}$ penicillin, $100 \mathrm{U} / \mathrm{ml}$ streptomycin for 1 day and 2 days. Bone marrow mesenchymal stem cells (BMSCs) were cultured in 96-well plate (Sigma). The supernatant was removed and replaced with the medial soaked with decellularized composite scaffold (the experimental group, $\mathrm{n}=5$ ) for culturing 1,2,3 days. Correspondingly, the control groups were cultured in the normal medial without decellularized hypopharynx with vascular pedicle for culturing 1, 2, 3 days. $10 \mu \mathrm{L}$ CCK-8 reagent (Sigma) were added to culture for 4h. The OD value at $450 \mathrm{~nm}$ was determined by a microplate absorbance reader (HBS-1096C Pro, Nanjing,China) to determine the cell viability in each well.

2.4 Surgical transfer to the greater omentum

The abdominal skin of the anesthetized rabbits was sterilized. A midline incision in the abdominal all exposed the greater omentum. The fresh samples and decellularized samples were explanted and placed on the greater omentum. Then the greater omentum was sutured as a capsule with 6-0 nylon silk sutures and abdominal was anastomosed. All animals were returned to their cages with no immunosuppressive agents administered and treated with penicillin for 3 days. Transplanted scaffolds were acquired from the greater omentum of receptor rabbits after 3, 8 and 12 weeks. For histological analysis, the sections were stained with HE and the operation referenced to the process above.

\subsection{Recellularization of decellularized hypopharynx}

\subsubsection{Isolation, culture and myogenic differentiation of BMSCs}

Twelve receptor rabbits (4-week-old rabbits) were were euthanized by intraperitoneal sodium pentobarbital administration $(100 \mathrm{mg} / \mathrm{kg})$. Three of them were used for immunocytochemistry and the remaining rabbits for recellularization. The legs were soaked in $70 \%$ ethanol for $5 \mathrm{~min}$. Then bone marrow was aspirated from the femurs in a sterile fashion. Bone marrow cells mixed with heparin were suspended and washed in PBS. Mononuclear cells were isolated by density gradient centrifugation (Histopaque $1.077 \mathrm{~g} / \mathrm{ml}$; Sigma). Cells were washed and finally resuspended in L-DMEM (1500 rpm, $10 \mathrm{~min}$; Sigma) and seeded at a density of $10^{6}$ cells $/ \mathrm{ml}$ in L-DMEM supplemented with $15 \%$ fetal bovine serum (FBS, Hyclone, USA), $100 \mathrm{U} / \mathrm{ml}$ penicillin, $100 \mathrm{U} / \mathrm{ml}$ streptomycin, and $2 \mathrm{~mol} / \mathrm{L} \mathrm{L-glutamine} \mathrm{(Sigma)} \mathrm{in} 100 \mathrm{~cm}^{2}$ petri dish. Cultures were maintained in a humidified atmosphere with $5 \%$ carbon dioxide at $37{ }^{\circ} \mathrm{C}$. The media was changed every 3 days. At $80 \%$ confluence, primary BMSCs were washed twice in Sterile Saline Solution and cultured with 5 -azacytidine $(10 \mathrm{mmol} / \mathrm{L}$; Sigma) for $24 \mathrm{~h}$ for differentiation into myocytes. Cells were digested with $0.25 \%$ trypsin-EDTA (Sigma) and passaged at the ratio of 1: 3 . Cells between passage 3 and passage 5 were used. Cells were examined by immunocytochemistry with antibody 
to a-sarcomeric actin. The receptor rabbits were provided with penicillin for 1 week and kept in a sterile environment postoperatively.

\subsubsection{Recellularized scaffolds}

The decellularized hypopharynx scaffold was placed in L-DMEM with 15\% FBS after perfusion. Cells were digested and cells $(80-100 \times 106)$ were suspended in $1 \mathrm{ml}$ L-DMEM with $15 \%$ FBS. They were injected multipointly in the decellularized matrix with a $20 \mathrm{ul}$ microinjection needle. 30 min later, cellular numbers were examined and quantified in the effluent from the decellularized matrix after injection. This recellularized scaffolds was immersed in L-DMEM with $15 \% \mathrm{FBS}$ in a humidified atmosphere with $5 \%$ carbon dioxide at $37^{\circ} \mathrm{C}$ for $24 \mathrm{~h}$.

\subsubsection{Histological analysis of recellularized scaffolds in vitro}

For histological analysis, SEM was used to examine the cells adhered to the decellularized hypopharynx scaffold and the operation referenced to the process above.

\subsubsection{Surgical transfer to the greater omentum}

The operation of recellularized hypopharyngeal explantation referenced to the process above and the recellularized scaffold was implanted on the greater omentum for 1 week.

2.5.5 Histology and immunohistochemistry of recellularized scaffolds in vivo

Histological examination was used to examine the cells on the scaffold. For histological analysis, the sections were stained with $\mathrm{HE}$ and the operation referenced to the process above. Immunohistochemistry was used to examine the signs of myogenic differentiation on the decellularized scaffolds. For immunohistochemistry analysis, sections were dewaxed and rehydrated, antigen repaire, perforated and incubated with a series of blocking solution. Following by, sections were exposed to the primary antibody to $\alpha$-sarcomeric actin (1: 200 dilution, mouse monoclonal antibody, skeletal muscle $\mathrm{Ab}-2$; Thermo, USA) for $1 \mathrm{~h}$ at $37^{\circ} \mathrm{C}$. Sections were washed in PBS and then exposed to the secondary antibody (1: 1000 dilution, goat antimouse IgG; China). Subsequently, sections were incubated with avidin/ horseradish peroxidase (HRP) for $30 \mathrm{~min}$ at $37^{\circ} \mathrm{C}$. Subsequently, the sections were stained with chromogenic substrate diaminobenzidine (DAB) for 5 minutes at room temperature.Sections were then counterstained with hematoxylin.

\section{Results}

\subsection{Characterization of the decellularized scaffold}

The images of the decellularized tissue indicated that it is efficient to remove the majority of the cellular element by perfusing detergent. After 16 hours of $1 \%$ SDS and triton X-100 perfusion, the decellularized scaffolds (Fig. 1B) were transparent and intact and there was no significant change in size compared to the fresh scaffolds macroscopically (Fig. 1A). HE staining indicated that there was only extracellular matrix and no cellular components in the decellularized hypopharynx (Fig. 1D) and ascending pharyngeal artery (Fig. 1F), while cellular components were found in the fresh hypopharynx (Fig. 1C) and ascending pharyngeal artery under light microscope (Fig. 1E).

SEM indicated that all cellular components were removed, but the ECMs were preserved. Collagen bundles can be observed on the surface of decellularized hypopharynx (Fig. 2B) and ascending pharyngeal artery scaffold (Fig. 2D), while collagen bundles and ECM cannot be seen in the native hypopharynx (Fig. 2A) and ascending pharyngeal artery with cellular components (Fig. 2C).

The average DNA content of the native hypopharynx and ascending pharyngeal artery 
samples was $1650 \mathrm{ng} / \mathrm{mg}$ dry weight versus $50 \mathrm{ng} / \mathrm{mmg}$ of decellularized hypopharynx dry weight and $42 \mathrm{ng} / \mathrm{mg}$ of decellularized vessel dry weight. The average DNA content of the decellularized hypopharynx and vessel samples were less than that in the fresh hypopharynx with vessel samples ( $\mathrm{P}<0.05$; Fig. 3). DNA analysis showed that the housekeeping gene ( $\beta$-actin) in the decellularized hypopharynx and ascending pharyngeal artery sample were not amplified.

3.2 Immunogenicity of the decellularized scaffold

Immunofluorescence staining indicated that the presence of the markers of MHC-II in fresh tissue can be observed (fluorescence on hypopharynx and ascending pharyngeal artery) (Fig. 4A). However, the markers of MHC-II were not present on the decellularized scaffold. Meanwhile, DAPI staining indicated that there were a mass of nuclei in the fresh hypopharynx and ascending pharyngeal artery. But no nuclei were found in the decellularized hypopharynx and ascending pharyngeal artery.The cells were eliminated completely by perfusion.

All the implanted fresh hypopharynx samples showed strong immunological rejection (Fig. 4B). The native scaffolds and decellularized samples were encapsulated in the greater omentum. HE staining indicated that there were extensive acute inflammatory cells infiltrating in the fresh samples, while slight acute inflammatory cells infiltrating in the decellularized samples implanted in the greater omentum after 3 weeks. Some lymphocytes and necrotic tissue were observed in the fresh samples after 8 weeks. However, fewer lymphocytes and neovascularization were observed in the decellularized samples after 8 weeks. When the samples were implanted after 12 weeks, the decellularized samples degraded completely and greater omentum became thicker and the fresh samples were not discovered and purulent secretion were observed.

3.3 Cytotoxicity of decellularized hypopharynx with vascular pedicle

CCK8 assay was examined the cytotoxicity of decellularized scaffold. As examined by the CCK8 assay, no significant differences were observed between the experimental and control groups for extracts of decellularized scaffold in terms of the proliferation of rabbit BMSCs (Figure 5) $(\mathrm{n}=6, \mathrm{P}>.05)$.

\subsection{Characterization of BMSCs and BMSCs induced by 5-azacytidine}

BMSCs had adhered to the bottom of the petri dish and the shape of cells was fusiform after 3 days of cell culture (Figure. 6A). After 3 weeks of induced culturing by 5 -azacytidine, cells still kept their fusiform shape, while the cell morphology were enlarged (Figure. 6B). Immunocytochemistry indicated that the expression of $\alpha$-sarcomeric was positive in the cells induced by 5 -azacytidine (Figure. $6 \mathrm{C}$ ), while the expression of $\alpha$-sarcomeric was negative in the cells without 5-azacytidine (Figure. 6D).

\subsection{Characterization of recellularized scaffolds}

SEM indicated that cells were polygonal in shape and adhered to the surface of recellularized scaffolds in vivtro (Fig. 7A). HE staining showed cycloidal and oversized cells like regenerated myocytes growed in the recellularized scaffold after implanted on the greater omentum for 1 week (Fig. 7B). And immunocytochemistry confirmed that the BMSCs differentiated into myocytes for positive expression of $\alpha$-sarcomeric in the recellularized scaffold(Fig. 7C).

\section{Discussion}

Hypopharyngeal squamous cell carcinoma (HSCC) is a common malignant tumor in head and neck and the main therapy was operation, companied by chemoradiotherapy (CRT). The range 
of hypopharyngotomy is too extensive to close pharyngeal defect primarily. The autogenous flaps and artificial biomaterials have been considered as an alternative materials nowadays to repair the hypopharyngeal defects. However, the native hypopharynx tissue structure contained mucosal layer, submucosa, muscle layer and serosal layer. The autogenous flaps and artificial biomaterials cannot imitate the native anatomicals tructure of hypopharynx. Meanwhile, the autogenous flaps to reconstruction will cause second injury to sick and frail patient and artificial biomaterials are lack of vascellum nourishing the tissue. We aimed to prepare an acellular, natural, three-dimensional (3D) biological hypopharynx with vascular pedicle scaffold by perfusion techniques to reconstruct laryngopharynx.

ECM is composed of around 300 different proteins and carbohydrates, which is mainly composed of collagens, proteoglycans, and glycoproteins (42-44). So ECM serves as a building support for cell growth in tissue and affect cell migration, lineage commitment, cellular behavior during disease progression, stem cell proliferation and differentiation $(24,45-47)$. SDS and triton X-100 can eliminate cellular component and preserve ECM efficiently $(48,49)$. In our study, an acellular, natural, 3D biological hypopharynx with vascular pedicle was prepared by perfusing $1 \%$ SDS for $16 \mathrm{~h}$ and triton X-100 for $30 \mathrm{~min}$. The decellularized composite scaffold was transparent macroscopically after perfusion. Histological staining and SEM showed that the hypopharyngeal native ECM structure was preserved including mucosal layer, submucosa, muscle layer and no residual cell component was found. The decellularization criteria are as follows: 1) $<$ $50 \mathrm{ng}$ of dsDNA per mg dry weight, 2) DNA fragment length $<200 \mathrm{bp}$, and 3) lack of visible nuclear material in tissue sections (28). PCR and agarose electrophoresis indicated that little nucleic acid was residual. The slighter immune rejection of decellularized composite scaffold in vivo compared to the fresh samples further verified that none of cells exist in decellularized composite scaffold. CCK-8 suggested that the medial soaked with decellularized composite scaffold had no toxic effects on cell proliferation. In conclusion, the decellularized hypopharynx with vascular pedicle scaffold in our study retained the native, full-thickness, complex structure and component of ECM and microvasculature, which has the potential to repair extensive and complex defect in clinic. However, the allogeneic acellular dermal matrix patchs are too simplex to repair complex defects. The artificial tissue-engineered complex scaffold like 3D print cannot imitate the native structure of ECM and lack native elaborate microvasculature nursing tissue for the method of overlay printing. Meanwhile, the artificial biomaterials are lack of native component of ECM. Moreover, the economic cost of artificial scaffold is too high to be used extensively. By contrast, the native tissue-engineered biomaterials are low-cost and easy available.

The immunogenicity of the decellularized hypopharynx with vascular pedicle scaffold was removed by infusing $1 \%$ SDS and triton X-100 and it has a great potential to be a native muscle by recellularization. The main histocompatibility complex (MHC) resulting in immunological rejection mainly present on the cellular surface, while the ECM has no immunogenicity (50). Immunofluorescence staining indicated that the presence of markers of MHC-II was negative on the decellularized scaffolds in the study. It suggested that no cells were found in the decellularized composite scaffold by detergent perfusion. And the immunological rejection of decellularized composite scaffold in the allogeneic rabbit greater omentum was weaker visibly than the native tissues. The immunocytes were fewer in the decellularized composite scaffold and greater omentum encapsulating the decellularized scaffolds compared with the fresh samples. And the 
decellularized scaffolds in the greater omentum degrade completely after 3 months. It indicated that decellularized composite scaffold prepared in the experiment had little immunogenicity and favorable biodegradability. The cartilage-preserving decellularized laryngeal scaffolds by the same method had no significant immune rejection after implanted in an allogeneic rat (39). The decellularized kidney yielding acellular scaffolds with vascular, cortical and medullary architecture, collecting system and ureters was prepared by perfusion and it was transplanted orthotopically in xenogeneic rat successfully by Ott.etc (27). The regenerated kidney produced urine and parenchymal bleeding or microvascular thrombus formation were not observed in bloodperfused vasculature of explanted regenerated kidneys (27). It suggested that acellular materials with little immunogenicity could be prepared by detergent purfusion for reconstructing organs or tissues.

Marrow mesenchyml stem cells (MSCs) are pluripotent progenitor cells that possess self-renewal and multi-directional differentiation (51). MSCs can differentiate into different kind of cells and be easily amplified in vitro (52). Recent years,some studies showed that MSCs have immunoregulatory bioactivity and the main immunological characteristics are low immunogenicity and high immunosuppressive ability (53-55). MSCs produced a large variety of paracrine to exert its immunoregulatory effects, rather than contact-dependent mediators (56). MSCs have attracted great attention for their regenerative activity and strong immunosuppressive property of MSCs over the decades. In our experiment, BMSCs adhered to and proliferated on the decellularized hypopharynx with vascular pedicle scaffold in vitro and vivo. Moreover, BMSCs are successfully induced into myocytes by cultured with 5 -azacytidine in the decellularized scaffolds. It suggested that the decellularized composite scaffold we prepared may have an positive effect on inducing BMSCs into myocytes. Ott et al. perfused the aorta with SDS and triton X-100 to prepare decellularized hearts with an acellular, perfusable vascular architecture, competent acellular valves and intact chamber geometry and under physiological load and electrical stimulation, constructs could generate a degree of pump function in recellularized heart (26). Vascularized islet organs were prepared by endothelializing acellular lung matrixes and the bio-fabricate islet released physiologic insulin and reduced hyperglycemia successfully when they were i supra-physiologic arterial glucose levels in vivo and ex vivo (57).

The hypopharyngeal reconstruction after hypopharyngectomy has been a challenge all the time. The common materials have inevitable defects. Autogenous flaps will cause second injury to patients and cannot repair the defect repeatedly when the flaps necrosed. The artificial scaffolds are lack of the component and structure of native ECM. The allogeneic acellular dermal matrix patchs are of no full-thickness, complex, microvascular ECM. However, in anatomical structure, the decellularized hypopharynx with vascular pedicle scaffold we prepared retained the hypopharyngeal full-thickness native structure of ECM. On a biochemical level, the decellularized composite scaffold retained the native component of ECM and little immunogenicity. In conclusion, the decellularized composite scaffold has an potential to be an alternative material to hypopharyngeal reconstruction. In future, we plan to epithelialize the mucosa and prepare the functional hypopharynx.

\section{Conclusions}

The defect of hypopharyngectomy of HSCC is extensive and there is not perfect patching materials at present. An acellular, natural, 3D biological decellularized hypopharynx with vascular pedicle scaffold as the substitute materials for hypopharyngeal reconstruction could be prepared 
by perfusing $1 \%$ SDS and triton X-100. This composite decellularized scaffold preserved the structure and components of the natural ECM and have little immunogenicity. The decellularized composite we prepared had good prospect in hypopharyngeal reconstruction as an allogeneic material.

Author Contributions:Nan Hou,Die Lv:ideals, design of methodology,creation and writing-original draft prepartion,Nan Hou:acquisition of the financial support for the project leading to this publication.Die Lv,XiaoLi Xu, YanQing Lu,JingZhi Li,Ying Tang:provision of study materials,specifically performing the experiments,data presentation,RuiNa Ma,Yun Zheng:verfication,reproducibility of results,supervision

Funding: This research was supported by a grant from the Foundation of the Sichuan Science and Technology Department, No. 19ZDYF0944.

\section{Declarations}

Ethics approval and consent to participate :This study was approved by Ethics Committee of Chengdu Medical College

Consent for publication :Not applicable.

Conflicts of Interest: The author declares no conflict of interest.

\section{References:}

1. Takes RP, Strojan P, Silver CE, Bradley PJ, Haigentz M, Wolf GT, et al. Current trends in initial management of hypopharyngeal cancer: the declining use of open surgery. Head \& neck. 2012.

2. Hall SF, Groome PA, Irish J, O'Sullivan B. The natural history of patients with squamous cell carcinoma of the hypopharynx. The Laryngoscope. 2008.

3. Kuo P, Sosa JA, Burtness BA, Husain ZA, Mehra S, Roman SA, et al. Treatment trends and survival effects of chemotherapy for hypopharyngeal cancer: Analysis of the National Cancer Data Base. Cancer-Am Cancer Soc. 2016.

4. Cooper JS, Porter K, Mallin K, Hoffman HT, Weber RS, Ang KK, et al. National Cancer Database report on cancer of the head and neck: 10-year update. Head \& neck. 2009.

5. Yeh DH, Sahovaler A, Fung K. Reconstruction after salvage laryngectomy. Oral Oncol. 2017.

6. Microvascular COTA. Salvage laryngectomy and laryngopharyngectomy: Multicenter review of outcomes associated with a reconstructive approach. Head \& neck. 2019.

7. Genden EM, Rinaldo A, Shaha AR, Bradley PJ, Rhys-Evans PH, Ferlito A. Pharyngocutaneous fistula following laryngectomy. Acta Oto-Laryngol. 2004.

8. Yu P, Hanasono MM, Skoracki RJ, Baumann DP, Lewin JS, Weber RS, et al. Pharyngoesophageal reconstruction with the anterolateral thigh flap after total laryngopharyngectomy. Cancer-Am Cancer Soc. 2010.

9. Fung K, Teknos TN, Vandenberg CD, Lyden TH, Bradford CR, Hogikyan ND, et al. Prevention of wound complications following salvage laryngectomy using free vascularized tissue. Head \& neck. 2007.

10. Yeh DH, Sahovaler A, Fung K. Reconstruction after salvage laryngectomy. Oral Oncol. 2017.

11. Azizzadeh B, Yafai S, Rawnsley JD, Abemayor E, Sercarz JA, Calcaterra TC, et al. Radial forearm free flap pharyngoesophageal reconstruction. The Laryngoscope. 2001. 
12. Ahmed A, Visavadia BG, Farrell R, Gilhooly M. Use of double skin paddle for pharyngoesophageal reconstruction using tubed radial forearm free flap. The British journal of oral \& maxillofacial surgery. 2014.

13. Ahmed A, Visavadia BG, Farrell R, Gilhooly M. Use of double skin paddle for pharyngoesophageal reconstruction using tubed radial forearm free flap. The British journal of oral \& maxillofacial surgery. 2014.

14. Scharpf J, Esclamado RM. Reconstruction with radial forearm flaps after ablative surgery for hypopharyngeal cancer. Head \& neck. 2003.

15. Yu P, Hanasono MM, Skoracki RJ, Baumann DP, Lewin JS, Weber RS, et al. Pharyngoesophageal reconstruction with the anterolateral thigh flap after total laryngopharyngectomy. Cancer-Am Cancer Soc. 2010.

16. Theogaraj SD, Merritt WH, Acharya G, Cohen IK. The pectoralis major musculocutaneous island flap in single-stage reconstruction of the pharyngoesophageal region. Plast Reconstr Surg. 1980.

17. Emerick KS, Herr MA, Deschler DG. Supraclavicular flap reconstruction following total laryngectomy. The Laryngoscope. 2014.

18. Sethi RKV, Kozin ED, Lam AC, Emerick KS, Deschler DG. Primary tracheoesophageal puncture with supraclavicular artery island flap after total laryngectomy or laryngopharyngectomy. Otolaryngology--head and neck surgery : official journal of American Academy of Otolaryngology-Head and Neck Surgery. 2014.

19. Khan MN, Rodriguez LG, Pool CD, Laitman B, Hernandez C, Erovic BM, et al. The versatility of the serratus anterior free flap in head and neck reconstruction. The Laryngoscope. 2017.

20. Choi Y, Kim TG, Jeong J, Yi H, Park JW, Hwang W, et al. 3D Cell Printing of Functional Skeletal Muscle Constructs Using Skeletal Muscle-Derived Bioink. Adv Healthc Mater. 2016;5(20):2636-45. http://doi.org/10.1002/adhm.201600483

21. Hui A, Hong P, Bezuhly M. Use of acellular dermal matrices in laryngotracheal and pharyngeal reconstruction: systematic review. The Journal of laryngology and otology. 2017.

22. He J, Tian Y, Wu P, Liao L, Quan H, Kang J, et al. Heterogeneous bovine acellular dermal matrix for mucosal repair in reconstructive surgery for laryngeal and hypopharyngeal carcinoma. Oncol Res Treat. 2015.

23. Li P, Li S, Yang X, Tang Q, He X, Wang S. Application of xenogenic acellular dermal matrix in reconstruction of oncological hypopharyngeal defects. European archives of oto-rhino-laryngology : official journal of the European Federation of Oto-Rhino-Laryngological Societies (EUFOS) : affiliated with the German Society for Oto-Rhino-Laryngology - Head and Neck Surgery. 2016.

24. Frantz C, Stewart KM, Weaver VM. The extracellular matrix at a glance. J Cell Sci. 2010.

25. Badylak SF, Freytes DO, Gilbert TW. Reprint of: Extracellular matrix as a biological scaffold material: Structure and function. Acta Biomater. 2015.

26. Ott HC, Matthiesen TS, Goh S, Black LD, Kren SM, Netoff TI, et al. Perfusion-decellularized matrix: using nature's platform to engineer a bioartificial heart. Nat Med. 2008.

27. Song JJ, Guyette JP, Gilpin SE, Gonzalez G, Vacanti JP, Ott HC. Regeneration and experimental orthotopic transplantation of a bioengineered kidney. Nat Med. 2013.

28. Crapo PM, Gilbert TW, Badylak SF. An overview of tissue and whole organ decellularization processes. Biomaterials. 2011.

29. Momtahan N, Sukavaneshvar S, Roeder BL, Cook AD. Strategies and processes to decellularize 
and recellularize hearts to generate functional organs and reduce the risk of thrombosis. Tissue engineering. Part B, Reviews. 2015.

30. Kitahara H, Yagi H, Tajima K, Okamoto K, Yoshitake A, Aeba R, et al. Heterotopic transplantation of a decellularized and recellularized whole porcine heart. Interact Cardiov Th. 2016.

31. Yagi H, Fukumitsu K, Fukuda K, Kitago M, Shinoda M, Obara H, et al. Human-scale whole-organ bioengineering for liver transplantation: a regenerative medicine approach. Cell Transplant. 2013.

32. Katsuki Y, Yagi H, Okitsu T, Kitago M, Tajima K, Kadota Y, et al. Endocrine pancreas engineered using porcine islets and partial pancreatic scaffolds. Pancreatology : official journal of the International Association of Pancreatology (IAP) ... [et al.]. 2016.

33. Inaba Y, Yagi H, Kuroda K, Kato J, Kawai Y, Kasai M, et al. Transplantation of a decellularized mitral valve complex in pigs. Surg Today. 2020.

34. Baptista PM, Siddiqui MM, Lozier G, Rodriguez SR, Atala A, Soker S. The use of whole organ decellularization for the generation of a vascularized liver organoid. Hepatology (Baltimore, Md.). 2011.

35. Ott HC, Clippinger B, Conrad C, Schuetz C, Pomerantseva I, Ikonomou L, et al. Regeneration and orthotopic transplantation of a bioartificial lung. Nat Med. 2010.

36. McKee R, Wingert R. Repopulating Decellularized Kidney Scaffolds: An Avenue for Ex Vivo Organ Generation. Materials. 2016;9(3):190. http://doi.org/10.3390/ma9030190

37. Uygun BE, Soto-Gutierrez A, Yagi H, Izamis M, Guzzardi MA, Shulman C, et al. Organ reengineering through development of a transplantable recellularized liver graft using decellularized liver matrix. Nat Med. 2010.

38. Zhang J, Hu ZQ, Turner NJ, Teng SF, Cheng WY, Zhou HY, et al. Perfusion-decellularized skeletal muscle as a three-dimensional scaffold with a vascular network template. Biomaterials. 2016.

39. Ma R, Li M, Luo J, Yu H, Sun Y, Cheng S, et al. Structural integrity, ECM components and immunogenicity of decellularized laryngeal scaffold with preserved cartilage. Biomaterials. 2013.

40. Hou N, Cui P, Luo J, Ma R, Zhu L. Tissue-engineered larynx using perfusion-decellularized technique and mesenchymal stem cells in a rabbit model. Acta Oto-Laryngol. 2010;131(6):645-52. http://doi.org/10.3109/00016489.2010.547517

41. Cannon RB, Houlton JJ, Mendez E, Futran ND. Methods to reduce postoperative surgical site infections after head and neck oncology surgery. The Lancet. Oncology. 2017.

42. Naba A, Clauser KR, Hoersch S, Liu H, Carr SA, Hynes RO. The matrisome: in silico definition and in vivo characterization by proteomics of normal and tumor extracellular matrices. Molecular \& cellular proteomics : MCP. 2012.

43. Hynes RO, Naba A. Overview of the matrisome--an inventory of extracellular matrix constituents and functions. Csh Perspect Biol. 2012.

44. Hoshiba T. Decellularized Extracellular Matrix for Cancer Research. Materials. 2019;12(8):1311. http://doi.org/10.3390/ma12081311

45. Arakelian L, Caille C, Faivre L, Corté L, Bruneval P, Shamdani S, et al. A clinical-grade acellular matrix for esophageal replacement. J Tissue Eng Regen M. 2019.

46. Philp D, Chen SS, Fitzgerald W, Orenstein J, Margolis L, Kleinman HK. Complex extracellular matrices promote tissue-specific stem cell differentiation. Stem cells (Dayton, Ohio). 2005. 
47. Xing H, Lee H, Luo L, Kyriakides TR. Extracellular matrix-derived biomaterials in engineering cell function. Biotechnol Adv. 2020.

48. Willemse J, Verstegen MMA, Vermeulen A, Schurink IJ, Roest HP, van der Laan LJW, et al. Fast, robust and effective decellularization of whole human livers using mild detergents and pressure controlled perfusion. Materials science \& engineering. C, Materials for biological applications. 2020.

49. Poornejad N, Momtahan N, Salehi ASM, Scott DR, Fronk CA, Roeder BL, et al. Efficient decellularization of whole porcine kidneys improves reseeded cell behavior. Biomedical materials (Bristol, England). 2016.

50. Crapo PM, Gilbert TW, Badylak SF. An overview of tissue and whole organ decellularization processes. Biomaterials. 2011.

51. Morigi M, Benigni A. Mesenchymal stem cells and kidney repair. Nephrology, dialysis, transplantation : official publication of the European Dialysis and Transplant Association European Renal Association. 2013.

52. Dominici M, Le Blanc K, Mueller I, Slaper-Cortenbach I, Marini F, Krause D, et al. Minimal criteria for defining multipotent mesenchymal stromal cells. The International Society for Cellular Therapy position statement. Cytotherapy. 2006.

53. Ayala-Cuellar AP, Kang J, Jeung E, Choi K. Roles of Mesenchymal Stem Cells in Tissue Regeneration and Immunomodulation. Biomol Ther. 2019.

54. Uccelli A, de Rosbo NK. The immunomodulatory function of mesenchymal stem cells: mode of action and pathways. Ann Ny Acad Sci. 2015.

55. Yang J, Liu F, Wang J, Cheng M, Wang S, Xu D. Mesenchymal stem cells and mesenchymal stem cell-derived extracellular vesicles: Potential roles in rheumatic diseases. World J Stem Cells. 2020.

56. Zhou Y, Day A, Haykal S, Keating A, Waddell TK. Mesenchymal stromal cells augment CD4+ and CD8+ T-cell proliferation through a CCL2 pathway. Cytotherapy. 2013.

57. Citro A, Moser PT, Dugnani E, Rajab TK, Ren X, Evangelista-Leite D, et al. Biofabrication of a vascularized islet organ for type 1 diabetes. Biomaterials. 2019. 


\section{Figures}
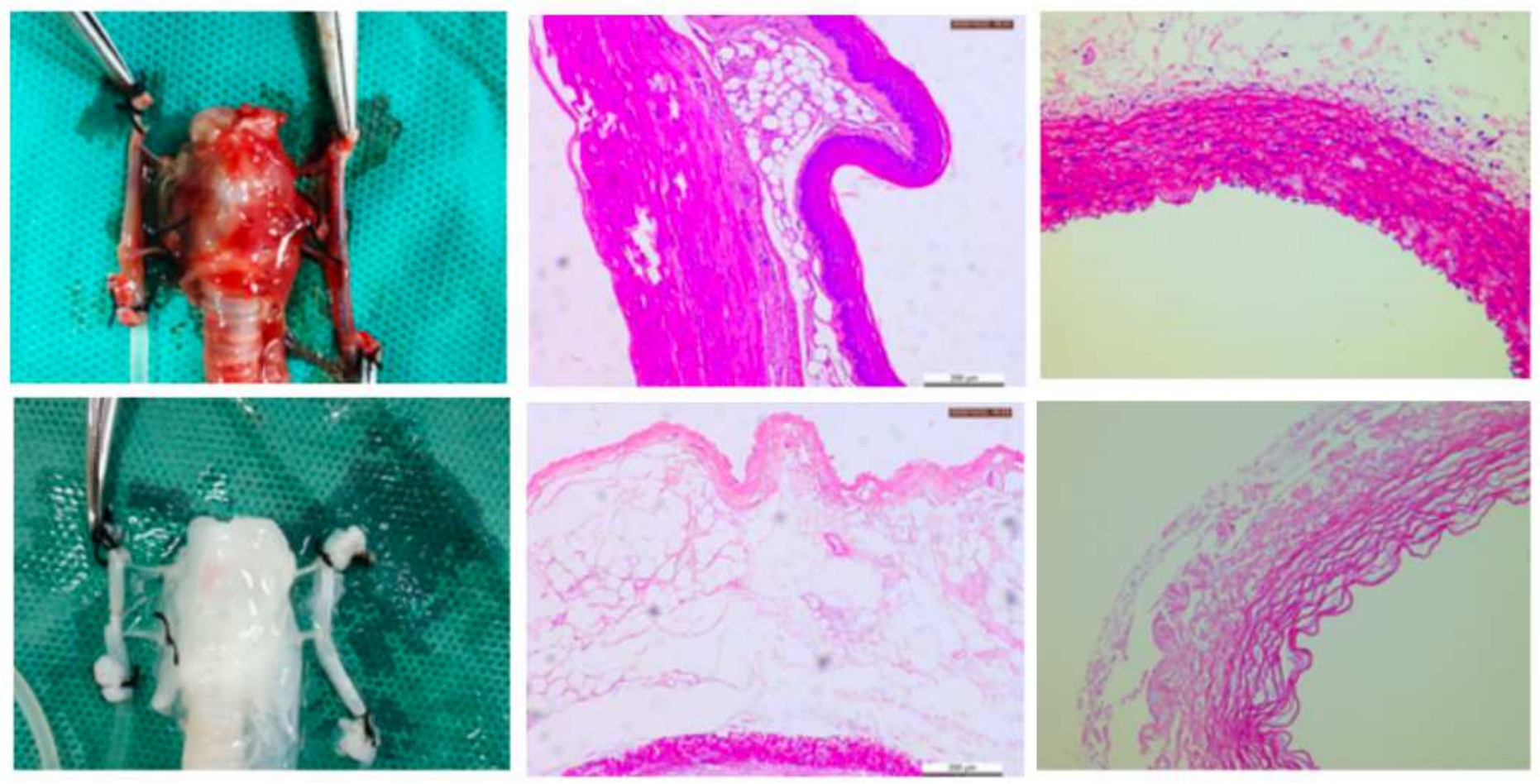

Fig. 1 Characterization of decellularized scaffold. Macroscopic appearance of fresh composite scaffold (A) including hypopharynx $(\rightarrow$ )and ascending pharyngeal artery macroscopically $(\boldsymbol{\Delta})$. Macroscopic appearance of composite decellularized scaffold (B) including hypopharynx $(\rightarrow)$ and ascending pharyngeal artery macroscopically $(\boldsymbol{\Delta})$. HE staining of the fresh hypopharynx (C), decellularized hypopharynx (D), fresh ascending pharyngeal artery (E) and decellularized ascending pharyngeal artery (F).There was no intact cells or nucleus in the ECM of the decellularized tissue (D, F).

Figure 1

Please See image above for figure legend. 

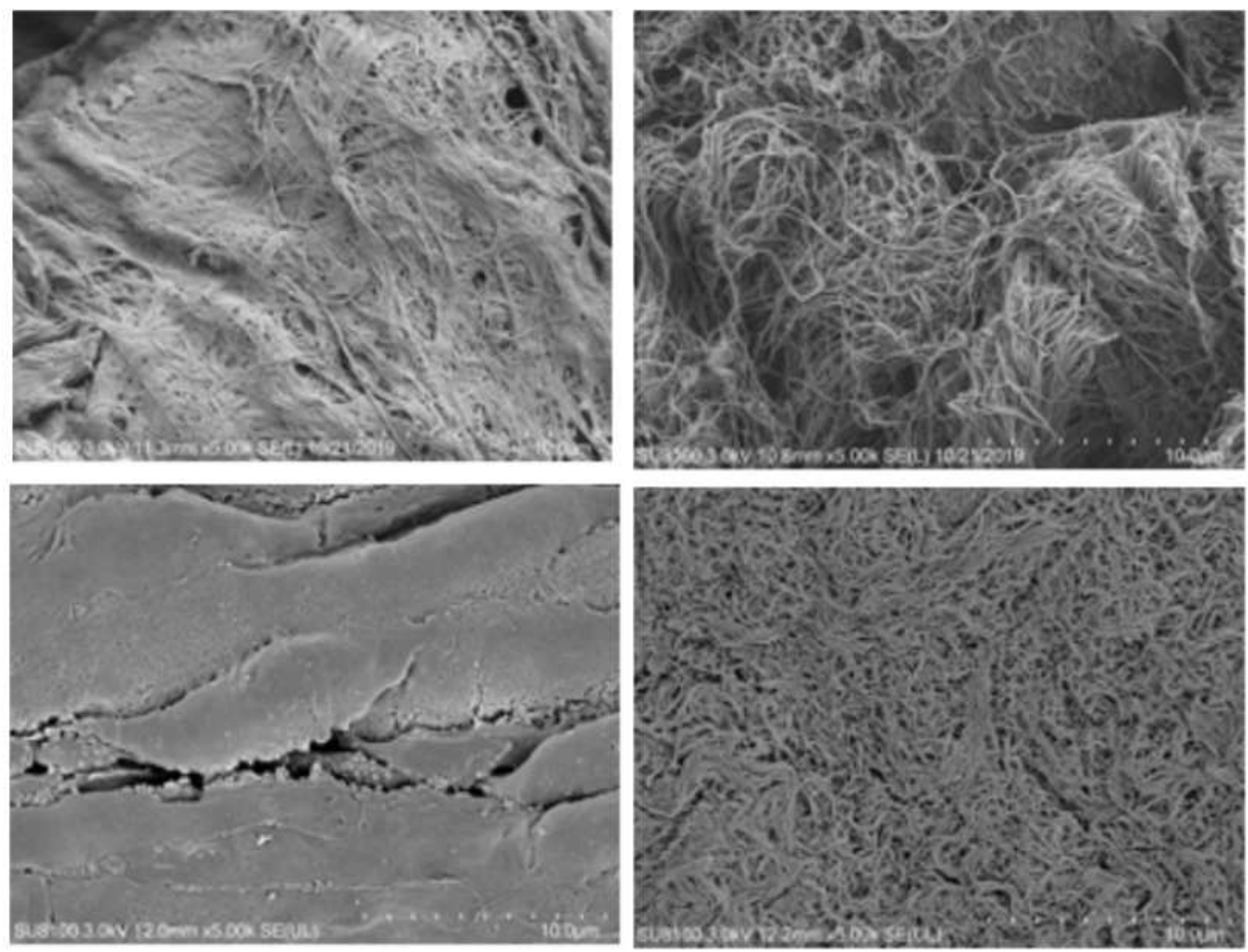

Fig. 2 Scanning electron micrographs (SEM) showed the fresh hypopharynx (A), fresh ascending pharyngeal artery (C), decellularized hypopharynx (B) and decellularized ascending pharyngeal artery (D). The collagen bundles was observed in the decellularized tissue (B, D).

Figure 2

Please See image above for figure legend. 
A

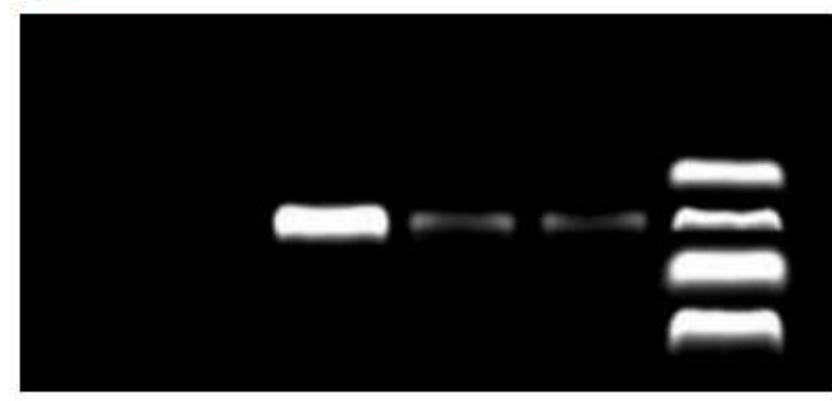

$100 \mathrm{bp}$
$250 \mathrm{bp}$
$500 \mathrm{bp}$
$750 \mathrm{bp}$

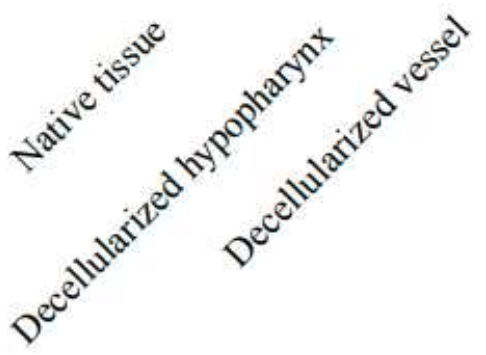

B

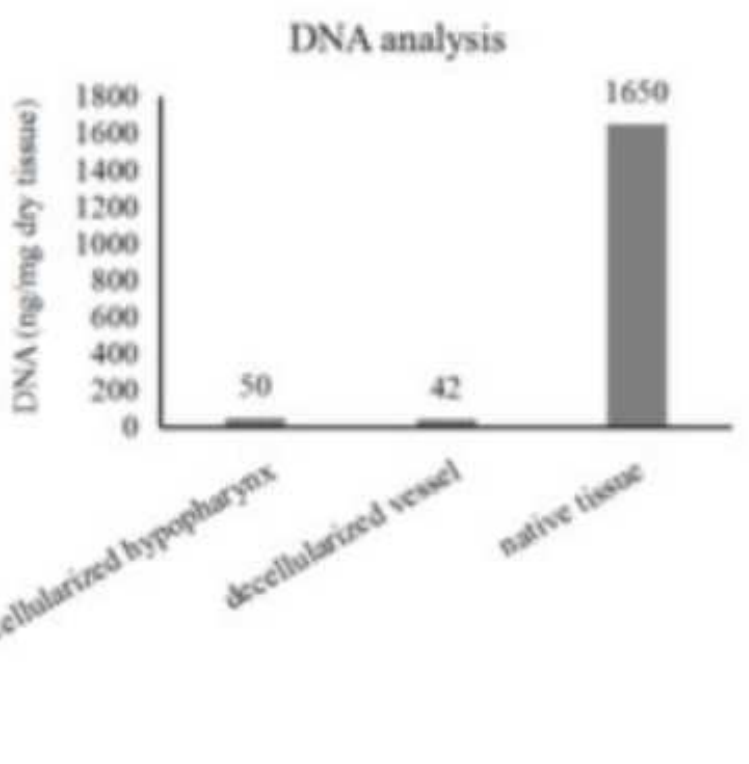

Fig. 3 PCR analysis of $\beta$-actin in extracts of the decellularized hypopharynx with vascular pedicle. Gel electrophoresis of PCR products for $\beta$-actin in ECM of the decellularized hypopharynx, decellularized ascending pharyngeal artery, and native hypopharynx with vascular pedicle (A). Bands represented respectively the residual of DNA content in ECM of decellularized hypopharynx, decellularized ascending pharyngeal artery, and native hypopharynx with vascular pedicle (B).

Figure 3

Please See image above for figure legend. 
A
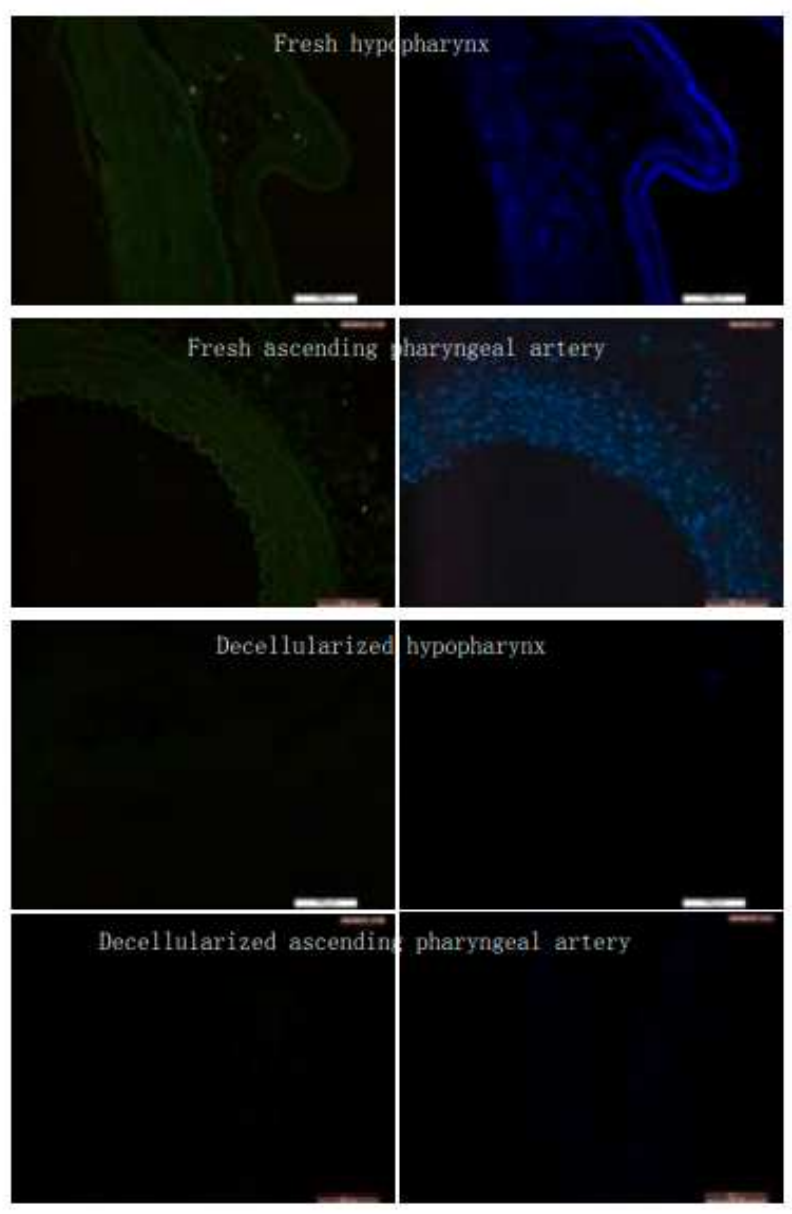

B
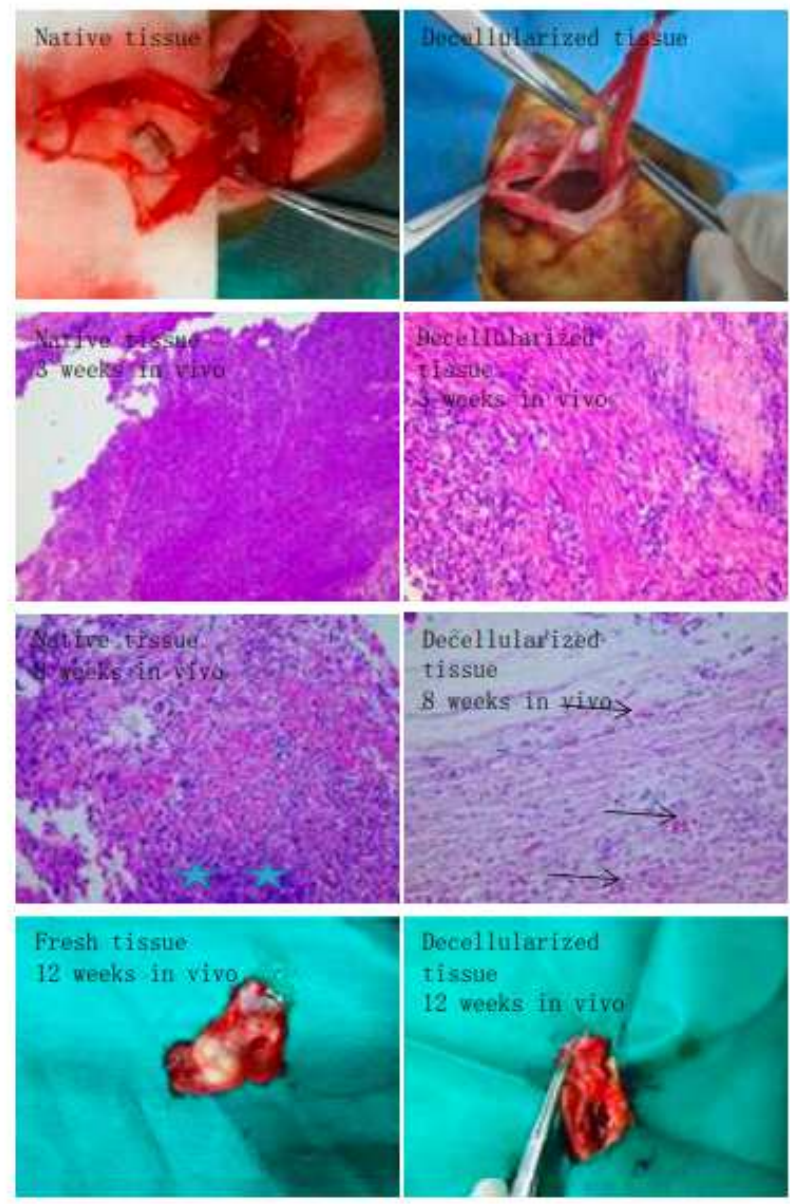

Fig. 4 Immunogenicity testing. Immunofluorescent staining showing that the presence of MHC-II antigens in the fresh tissue, low immunoreactivity in the decellularized tissue, and DAPI staining indicated that there were a mass of nuclei in the fresh scaffold, none of nuclei in the decellularized scaffold (A). Characterization of the implanted scaffolds in vivo (B). HE staining showed lots of immunocytes infiltrating in the fresh samples, and few immunocytes infiltrating in the decellularized samples after 3 weeks. The structure of native hypopharynx was destroyed and some necrotic tissue $(\hbar)$ were observed after 8 weeks. However,the structure of ECM in the decellularized hypopharynx was unbroken and neovascularization $(\rightarrow)$ were observed. Thicker greater omentum was seen compared with the native in the fresh samples and purulent secretion in the fresh samples after 12 weeks.

Figure 4

Please See image above for figure legend. 


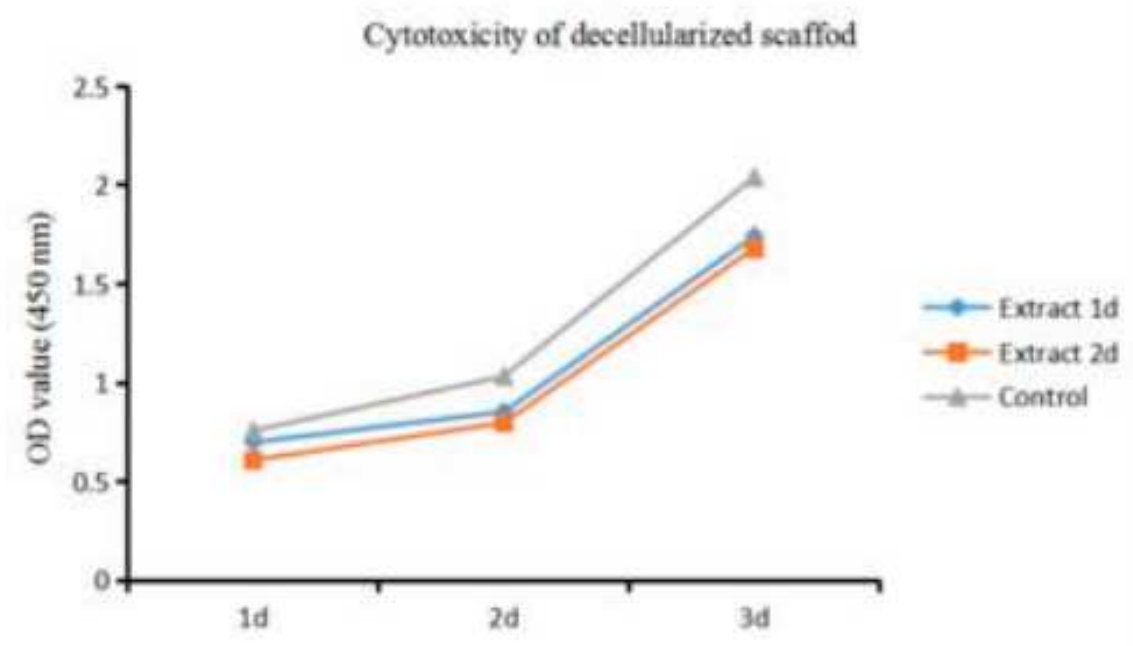

Fig. 5 Cytotoxicity assay. CCK8 assay for cytotoxicity examination of the scaffolds: the extract of ECM in decellularized hypopharynx with vascular pedicle scaffold (the medial soaked with decellularized composite scaffold for culturing 1,2 days) and well plate alone with OD values, $\mathrm{n}=5, \mathrm{P}>0.05$. The cells were cultured with the extract for 24,48 , and $72 \mathrm{~h}$.

Figure 5

Please See image above for figure legend. 



Fig. 6 Characterization of cells. BMSCs were cultured for 3 days (A). Cells were induced by 5 -azacytidine for 3 weeks (B). Immunocytochemistry showed the positive expression of $\alpha$-sarcomeric in the cells induced by 5 -azacytidine for 3 weeks $(\mathrm{C})$ and the negative expression in the cells without 5 -azacytidine for 3 weeks (D).

Figure 6

Please See image above for figure legend. 

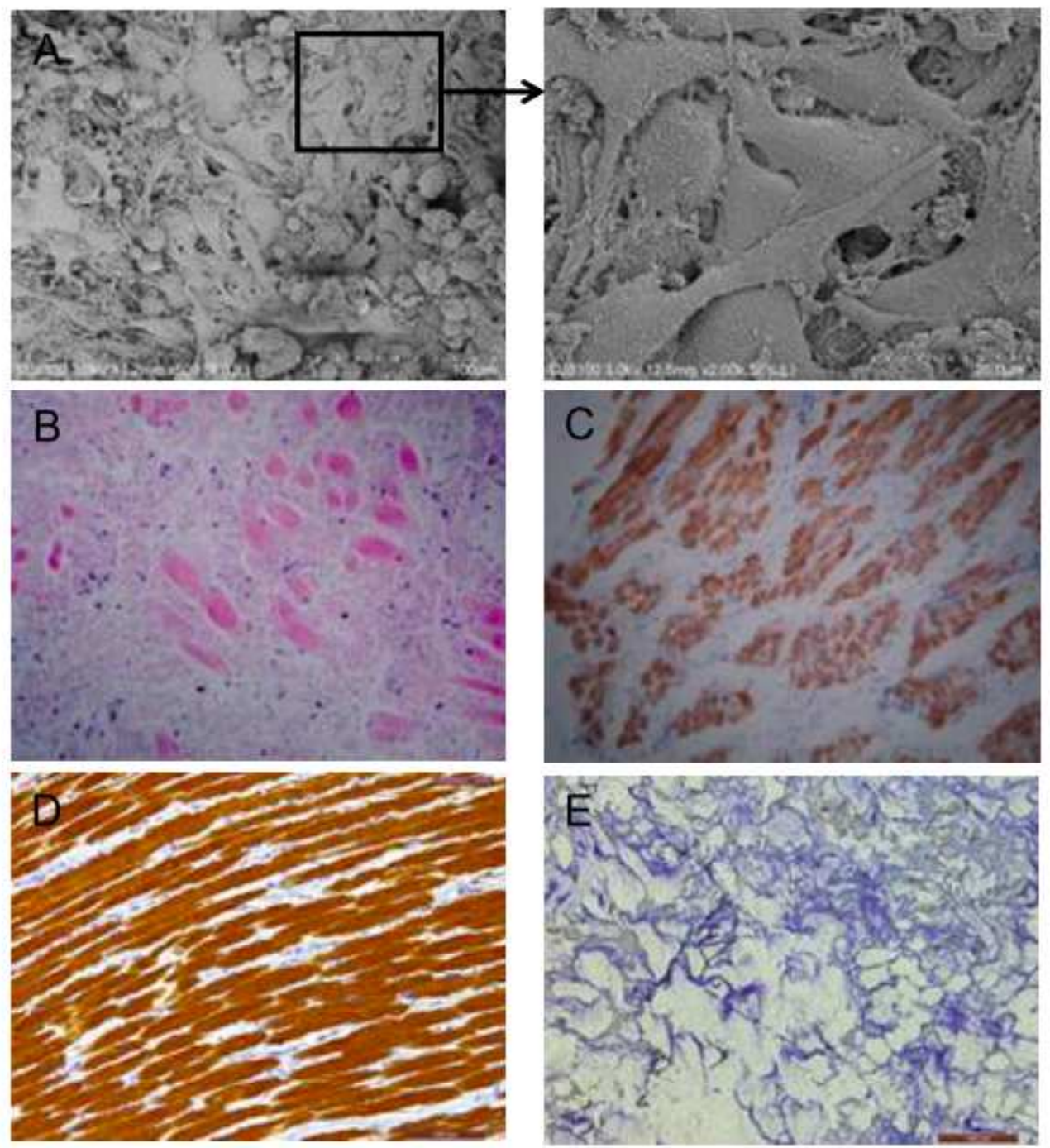

Fig. 7 Characterization of recellularized scaffolds. SEM showed cells adhered to the decellularized scaffolds (A). HE staining showed cycloidal and oversized cells like regenerated myocytes (B) growed in the recellularized scaffold after implanted on the greater omentum for 1 week. And immunocytochemistry confirmed that the cells were myocytes for positive expression of $\alpha$-sarcomeric in the recellularized scaffold(C). The abundant $\alpha$ sarcomeric were showed in the fresh hypopharynx (D) and little in the decellularized hypopharynx (E) by immunocytochemistry.

Figure 7

Please See image above for figure legend. 